\title{
Repulsion between molecules on a metal: Monolayers and submonolayers of hexa-peri-hexabenzocoronene on $\mathrm{Au}(111)$
}

\author{
C. Wagner, ${ }^{1,2, *}$ D. Kasemann, ${ }^{1}$ C. Golnik, ${ }^{1}$ R. Forker,,${ }^{1,3}$ M. Esslinger, ${ }^{1}$ K. Müllen, ${ }^{4}$ and T. Fritz ${ }^{1,3}$ \\ ${ }^{1}$ Institut für Angewandte Photophysik, Technische Universität Dresden, 01062 Dresden, Germany \\ ${ }^{2}$ Institut für Bio- und Nanosysteme 3, Forschungszentrum Jülich, 52425 Jülich, Germany \\ ${ }^{3}$ Institut für Festkörperphysik, Friedrich-Schiller-Universität Jena, 07743 Jena, Germany \\ ${ }^{4}$ Max-Planck-Institut für Polymerforschung, Ackermannweg 10, 55172 Mainz, Germany
}

(Received 19 December 2008; revised manuscript received 30 December 2009; published 25 January 2010)

\begin{abstract}
We investigate the growth of hexa-peri-hexabenzocoronene (HBC) on $\mathrm{Au}(111)$ for monolayer (ML) and sub-ML coverage by scanning tunneling microscopy and low-energy electron diffraction. A transition from a disordered isotropic phase at low coverage to a highly ordered phase with a coverage-dependent lattice constant at higher coverage is found and attributed to a repulsive intermolecular force. To deduce the origin of this repulsion a model is set up, containing the Coulomb interaction between molecules and between localized dipoles from the push-back effect, as well as the intermolecular van der Waals potential. The modeling of the van der Waals interaction is done on a force field level. We find that the observed repulsion can only be explained when assuming a certain screening of the attractive London forces by the presence of the metal substrate.
\end{abstract}

DOI: 10.1103/PhysRevB.81.035423

PACS number(s): 68.43.Fg, 81.15.Aa, 68.55.am, 61.05.jh

\section{INTRODUCTION}

The last years have witnessed a large increase in the research efforts on metal-organic interfaces, either in the context of "organic electronics" (organic light emitting diodes, organic photovoltaic devices, or organic field effect transistors), or in the context of future applications referred to as "molecular electronics." Especially the latter case requires a detailed understanding of highly ordered growth processes on metals as well as methods to design and structure more elaborate organic-organic heterojunctions. Hexa-peri-hexabenzocoronene (HBC) is a large conjugated planar hydrocarbon that is often considered as a model system in the context of organic electronics. ${ }^{1-5}$ While, in a closed monolayer (ML), HBC mostly grows planar in a hexagonal arrangement, recently also studies of $\mathrm{HBC}$ on $\mathrm{Au}(111)$ at very low coverage have been performed showing a nonplanar adsorption at step edges in face-centered-cubic regions. ${ }^{6,7}$ In addition, HBC on $\mathrm{Au}(111)$ has also been used as template for the heteroepitaxial growth of different organic species. ${ }^{2,8,9}$ While previous publications found a ML growth of HBC on $\mathrm{Au}(111)$ describable by a set of fixed lattice parameters, ${ }^{1-3}$ we reinvestigate the growth mechanism here, focusing especially on sub-ML coverage. Our experimental observation of a variable lattice constant, ascribed to an intermolecular repulsion is outlined in Sec. III. Subsequently, a model for the intermolecular potential is introduced, explicitly including attractive van der Waals forces (Sec. IV). The respective model calculations are outlined and discussed in Sec. V.

\section{EXPERIMENTAL METHODS}

Both, sample preparation and characterization were completely carried out under ultra-high vacuum conditions at room temperature. Thin HBC films of variable thickness up to $1 \mathrm{ML}$ are produced by organic molecular-beam epitaxy at an evaporation temperature of $\gtrsim 430{ }^{\circ} \mathrm{C}$ (Ref. 10) and a rate of $0.06 \mathrm{ML} / \mathrm{min}$. The molecular flux was initially monitored with a quartz-crystal microbalance, and was subsequently refined from low-energy electron diffraction (LEED) and scanning tunneling microscopy (STM) data of closed MLs. The substrate, an $\mathrm{Au}(111)$ single crystal, was cleaned by $\mathrm{Ar}^{+}$ sputtering and annealing cycles.

LEED images were acquired with a commercial four-grid display-type LEED setup (Omicron) and analyzed using a commercially available software. ${ }^{11}$ The displayed primary electron-beam energy was corrected according to a method proposed by Günther. ${ }^{12}$ Corrected values $E_{\text {corr }}$ are given here.

The commercial STM-1 (Omicron) operating at room temperature was used to record the STM images. Only drift correction but no smoothing or filtering was performed, the software WSXM (Ref. 13) was used to tune the image contrast.

\section{EXPERIMENTAL RESULTS}

Four exemplary LEED patterns obtained for varying coverage of $\mathrm{HBC}$ on $\mathrm{Au}(111)$ are compared in Fig. 1. We observe only diffuse rings for a coverage below $\approx 0.7 \mathrm{ML}$ [Figs. 1(a) and 1(b)]. Such a diffraction pattern is characteristic for an isotropic distribution of scatterers, separated by a certain preferred distance [we find separations of $(23 \pm 2)$ and $(19 \pm 1) \AA$ for Figs. 1(a) and 1(b), respectively]. At a coverage between 0.7 and $0.8 \mathrm{ML}$, however, ordering sets in as the diffuse rings coexist with rather broad spots indicating molecular islands in a hexagonal arrangement at a fixed domain angle $\delta$. In Fig. 1(c) the lattice constant is $a$ $=(15.9 \pm 0.2) \AA$, while Fig. 1(d) shows sharp LEED spots with $a=(14.6 \pm 0.1) \AA$ at a coverage of 0.95 ML. The definition of a ML coverage used throughout this paper refers to the highest density of HBC molecules observed in a closed layer which equals 0.57 molecules per $\mathrm{nm}^{2}$ at a lattice constant of $14.2 \AA$. A detailed investigation of the growth of HBC from 0.8 up to $1.0 \mathrm{ML}$ shows systematic variations of 


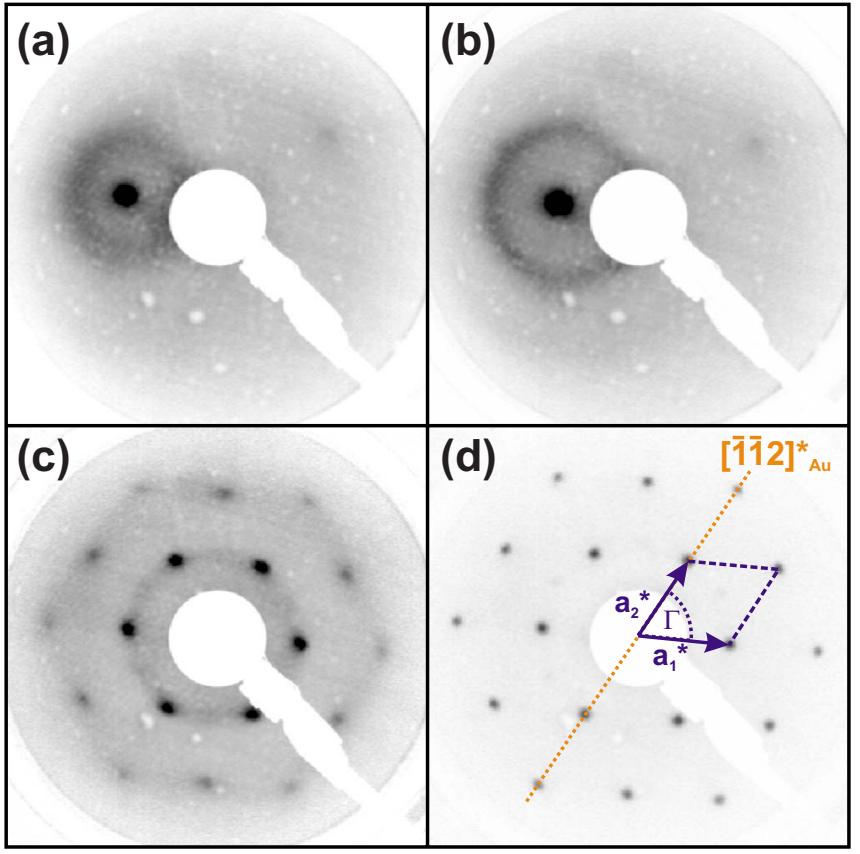

(e) POL

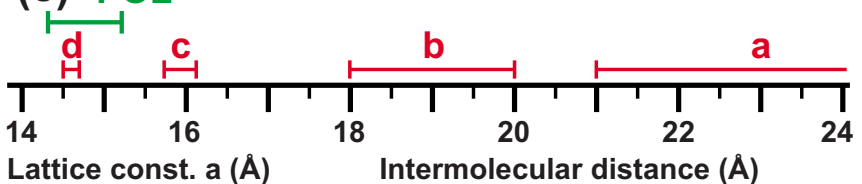

FIG. 1. (Color online) LEED patterns for varying HBC coverage. All images have been recorded at $E_{\text {corr }}=10.4 \mathrm{eV}$. (a) $(0.4 \pm 0.05)$ ML of HBC, off-center, extracted intermolecular distance $(23 \pm 2) \AA$. (b) $(0.6 \pm 0.05) \mathrm{ML}$ of HBC, off-center, extracted intermolecular distance $(19 \pm 1) \AA$. (c) $(0.8 \pm 0.05) \mathrm{ML}$ of $\mathrm{HBC}$, extracted lattice constant $a=(15.9 \pm 0.2) \AA$. (d) $(0.95 \pm 0.05) \mathrm{ML}$ of HBC, extracted lattice constant $a=(14.6 \pm 0.1) \AA$. The reciprocal unit cell $\left(\Gamma=(60 \pm 1)^{\circ}\right)$ is shown in blue. The domain orientation is $\delta=(0 \pm 1)^{\circ}$ vs the $[\overline{1} 12]_{\mathrm{Au}}$ direction. (e) Summary of the lattice constants (error bars in red). The region where, in principle, pointon-line growth could occur, is indicated.

the lattice constant $a$ : with increasing coverage, $a$ decreases from $\approx 16 \AA$ (where the ordered phase still coexists with disordered molecules) to a minimum of $(14.2 \pm 0.1) \AA$.

A similar behavior has been observed before for other molecule-metal combinations, which was ascribed to a lack of intermolecular interaction ${ }^{4,14}$ or even repulsive interactions ${ }^{15-17}$ without further explanation. Remarkably, most of the molecules for which repulsion was discussed are aromatic hydrocarbons without heteroatoms, for example perylene, coronene, naphthalene, para-hexaphenyl, and paraquaterphenyl. Recently, a repulsive interaction was reported for the donor molecule tetrathiafulvalene (TTF) on $\mathrm{Au}(111)$, and the charge transferred to the substrate was calculated by density-functional theory (DFT). ${ }^{18}$ Similarly, the intermolecular repulsion in sub-ML films of the phthalocyanine $(\mathrm{Pc})$ molecules $\mathrm{SnPc}$ and $\mathrm{CuPc}$ on $\mathrm{Ag}(111)$ could be linked to a charge donation/backdonation effect. ${ }^{19}$ One aspect of our contribution is to demonstrate that the observed repulsion in sub-ML HBC films on $\mathrm{Au}(111)$ can finally explain the dis-

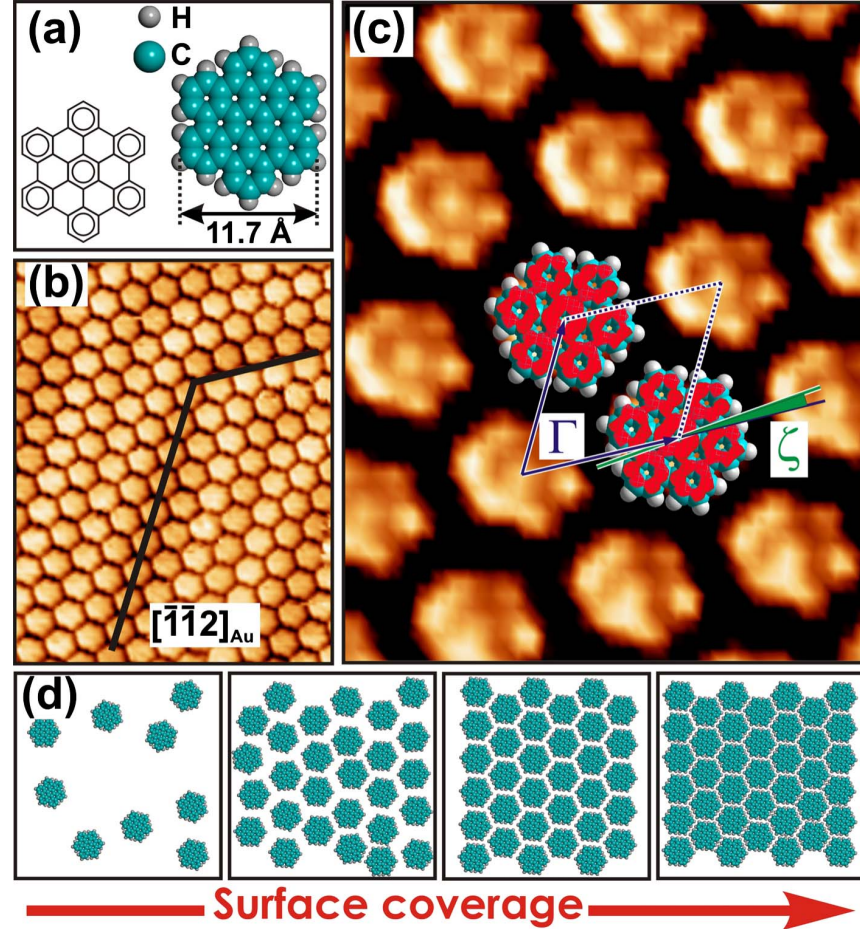

FIG. 2. (Color online) (a) Skeletal formula and a space-filling model of HBC. (b) STM image $\left(15 \times 19 \mathrm{~nm}^{2}, \mathrm{~V}=-1.4 \mathrm{~V}, I\right.$ $=75 \mathrm{pA})$ of a $\mathrm{HBC}$ ML on $\mathrm{Au}(111)$ with indicated $[\overline{1} \overline{1} 2]_{\mathrm{Au}}$ direction. (c) Closeup view of (b) $\left(4.5 \times 6.0 \mathrm{~nm}^{2}\right)$ with enhanced contrast. Orientation and size of two HBC molecules highest occupied molecular orbital [(HOMO) shown in red] are adjusted to match the STM contrast. The deduced angle between the short axis of HBC and a primitive lattice vector a is $\zeta=(5 \pm 1.5)^{\circ}$. (d) Schematic model of the molecular growth under a repulsive potential.

crepancies in the published data on the lattice constant and the epitaxial relation in this system. ${ }^{1-3}$

For an attractive intermolecular interaction small islands are evidently formed already at low coverage. In contrast, under the influence of a repulsive interaction no molecular lattice is formed up to a relatively high coverage at which the intermolecular distances decrease such that free molecular rotation is sterically hindered, as schematically depicted in Fig. 2(d). According to our experimental results there is a transitional range for the lattice formation at lattice constants in between $15<a<17 \AA$, with a coexistence of ordered and disordered regions at $a \approx 16 \AA$. At this coverage a "prelattice" is formed within which the molecules are still quite mobile. Upon ongoing deposition, the intermolecular distances are further reduced, finally forcing the molecules into a regular lattice.

In order to model this behavior quantitatively, detailed information on the structure of the HBC film is necessary. While the unit-cell parameters $a$ and $\Gamma=(60 \pm 1)^{\circ}$ are derived from LEED, the angle $\zeta$ between the short symmetry axis of HBC and a primitive lattice vector is deduced by analyzing the STM image in Fig. 2(c). It shows an enlarged and contrast-enhanced part of Fig. 2(b) and exhibits an intramolecular structure corresponding to the HOMO of HBC. The analysis yields $\zeta=(5.0 \pm 1.5)^{\circ}$, a value that allows adja- 
cent $\mathrm{H}$ atoms to interlock as illustrated by two model molecules.

In the following, we want to clarify whether the observed repulsion with a continuous change of the lattice constant (especially in the range between 14.2 and $\approx 16 \AA$ ) can be understood as the result of repulsive Coulomb forces originating from charge redistribution localized at the $\mathrm{HBC}$ adsorption sites as suggested before for other molecules. ${ }^{16,18}$ Here, the central question is whether the Coulomb repulsion actually dominates the attractive van der Waals forces.

\section{MODELING TECHNIQUE}

In our model, the intermolecular interaction consists basically of two parts: first, the Coulomb interaction due to partial charges on the molecules and due to localized dipoles resulting from the cushion or push-back effect, and second the van der Waals forces consisting of attractive London and repulsive Pauli forces. In the following these contributions will be discussed separately.

The charge distribution in the HBC molecule (relevant for the intermolecular Coulomb potential) is calculated for a free HBC molecule by the semiempirical PM3 method (using a commercial software ${ }^{20}$ ) assigning a partial charge to each atom. The Coulomb potential between two molecules is then calculated as the sum over all atom-atom pair potentials. As UPS measurements reveal a low density of states at the Fermi level of a $\mathrm{HBC} \mathrm{ML}$ on $\mathrm{Au}(111),{ }^{1}$ we conclude a mainly physisorptive binding of HBC to the $\mathrm{Au}(111)$ surface. In turn, this leads to the assumption that the electronic structures of both, the adsorbate and the substrate, are only weakly perturbed. Consequently, the use of charge distributions calculated for an isolated HBC molecule in vacuum should be sufficiently correct. The presence of the metal is accounted for by the introduction of mirror charges. According to Ref. 21, the effective location of the metal surface in the presence of an external charge is $1.94 \AA$ above the terminal lattice plane of $\mathrm{Au}(111)$. As no data for the precise adsorption height of $\mathrm{HBC}$ on $\mathrm{Au}(111)$ is available, we choose the value measured for the well studied system of 3,4,9,10perylenetetracarboxylic dianhydride (PTCDA) on Au(111) where a value of $3.27 \AA$ was found. ${ }^{22}$ We thus end up with a distance of $2.66 \AA$ between charge and image charge. The accuracy of this value is hard to estimate, which is, nevertheless, acceptable as the influence of the mirror charges is rather weak for the intermolecular separations found in an ordered HBC film $(14.2<a<16 \AA)$.

The push-back effect occurs upon adsorption of planar molecules on metals when the electron density spilling out of the bare surface is pushed back, forming a dipole perpendicular to the surface that is localized below the molecule, according to several corresponding theoretical calculations. ${ }^{18,23}$ We model this effect by a circular plate capacitor using the following parameters that are in accordance with DFT calculations: ${ }^{23}$ the plate separation is $l=2 \AA$ and the charge density on each plate is $\rho=0.3 \mathrm{e} / \mathrm{nm}^{2}$. We would like to note that the value for $l$ is not identical to the value that the authors themselves give for a plate capacitor model in Ref. 23. This discrepancy is due to the fact that Rusu et al. construct a capacitor to model (and match) the interface dipole while in our case the actual charge distribution is relevant. The diameter of the capacitor is given by the size of the molecule. We choose the upper limit of $d=12 \AA$, according to the dimensions given in Fig. 2(a). In doing so we probably overestimate the influence of this "push-back dipole" as calculations in Ref. 23 show that the push-back effect manifests itself especially in the region of the aromatic system. Although the above assumptions constitute a rather basic model, our results do not depend on a high accuracy of this particular component as will be shown in the next section.

We use an atomic force field approach to model the intermolecular van der Waals forces. The calculation is carried out by means of the software POWERGRID (Ref. 24) using the algorithm outlined in Ref. 25. The nonbonding optimized potentials for liquid simulations (OPLS) parameter set ${ }^{26}$ developed especially for the interaction between hydrocarbons is used to parameterize the respective Lennard-Jones (LJ) potentials. The use of this empirical method is motivated by the fact that also in calculations on a higher theoretical level, such as DFT, the very important nonbonding dissipative van der Waals forces are usually treated by a semiempirical dispersion correction. Only recently, first steps were made to overcome this limitation, ${ }^{27}$ however, only for small benzenelike molecules and for the short interaction distance between molecule and metal substrate and not between different molecules. The method used here, on the other hand, is able to reproduce the adsorption energy per molecule in a $\mathrm{HBC}$ multilayer system ${ }^{28} \quad[(-2.2 \pm 0.1) \mathrm{eV}$ from thermaldesorption spectroscopy (TDS) data $\left.{ }^{29}\right]$ within an accuracy of $10 \%$ (not shown).

Complete negligence of the molecule-surface interaction as done in this paper cannot be justified in general, but only for the present lattice/molecule orientation of $\mathrm{HBC}$ on $\operatorname{Au}(111)\left(\delta=(0 \pm 1)^{\circ}\right)$. Usually, one would have to consider local minima in the molecule-substrate potential, i.e., epitaxial structures such as commensurate or point-on-line epitaxy. ${ }^{25}$ For the observed HBC lattice, however, such epitaxial growth is possible only in a small lattice-constant region [green bar in Fig. 1(e)], and even there the energetic gain related to the epitaxial structures is almost negligible according to potential-energy calculations that will be published elsewhere.

\section{MODEL RESULTS AND DISCUSSION}

The Coulomb potential calculated as a function of the intermolecular distance is shown in Fig. 3. The calculation is conducted for two HBC molecules oriented at the observed angle $\zeta=5^{\circ}$. To illustrate a key issue, a similar calculation is also performed for a different molecular species with a dissimilar behavior: two PTCDA molecules in a geometry resembling the unit cell of PTCDA on $\mathrm{Au}(111) .{ }^{30}$ While a variation of the molecular separation $a$ is meaningful for HBC (observed in the experiment), for PTCDA this is only done to illustrate the behavior of the attractive Coulomb potential. Primarily relevant is only the potential at $a=11.5 \AA$, resembling the distance of the two molecules in the PTCDA 


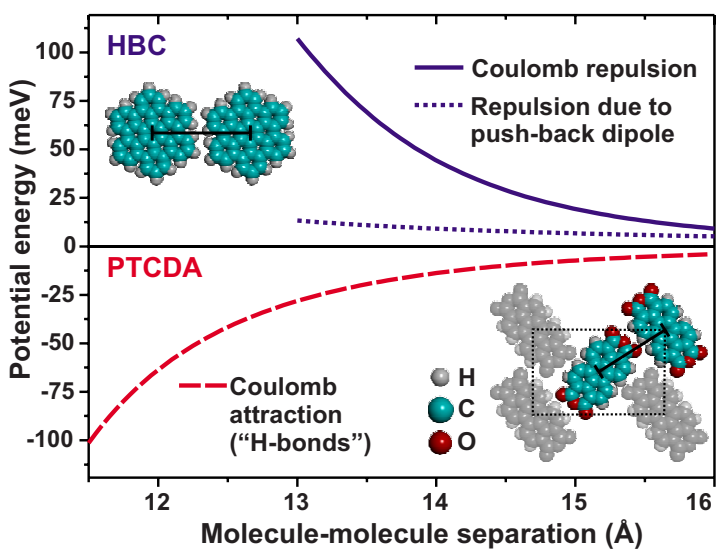

FIG. 3. (Color online) Separation-dependent Coulomb potential between a pair of two HBC molecules (solid blue), of two PTCDA molecules in the herringbone geometry (dashed red), and of two circular push-back dipole plate capacitors $(l=2 \AA, d=12 \AA, \rho$ $=0.3 \mathrm{e} / \mathrm{nm}^{2}$ ) (dotted blue).

unit cell. One immediately realizes that the intermolecular Coulomb potential is dominated by the repulsion or attraction between adjacent atoms in both molecules, i.e., repulsion between positively charged $\mathrm{H}$ atoms in the case of HBC and attraction between $\mathrm{H}$ atoms and negatively charged oxygen atoms ("hydrogen bond") in the case of PTCDA. This is illustrated in Fig. 4 where the partial charges of HBC and PTCDA are plotted. The influence of the push-back dipole (calculated for HBC only) is, in contrast, comparably weak. This first result coincides well with the fact that a repulsive interaction in a sub-ML has been observed for molecules without heteroatoms in the outer rim only, while, for example, PTCDA grows in islands at sub-ML coverage. ${ }^{31}$ The case of TTF on $\mathrm{Au}(111)$ (Ref. 18) is slightly different, as there the charge density (in a plate capacitor model) would be much higher due to the donor character and the small size of this molecule.

The question, whether the total Coulomb repulsion between the HBC molecules can account for the observed behavior on $\mathrm{Au}(111)$ is addressed in Fig. 5. Here, the POWERGRID calculation is performed for a HBC ML with a hexagonal unit cell and, again, as a function of the lattice constant $a$. Consequently, the energies (per molecule) deviate

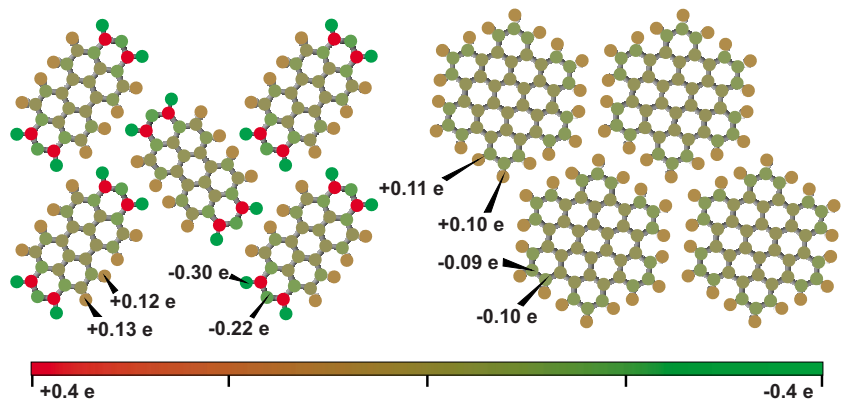

FIG. 4. (Color online) Partial charges of PTCDA and HBC as calculated for single molecules in vacuum by the semiempirical PM3 method. The most relevant partial charges are explicitly indicated.

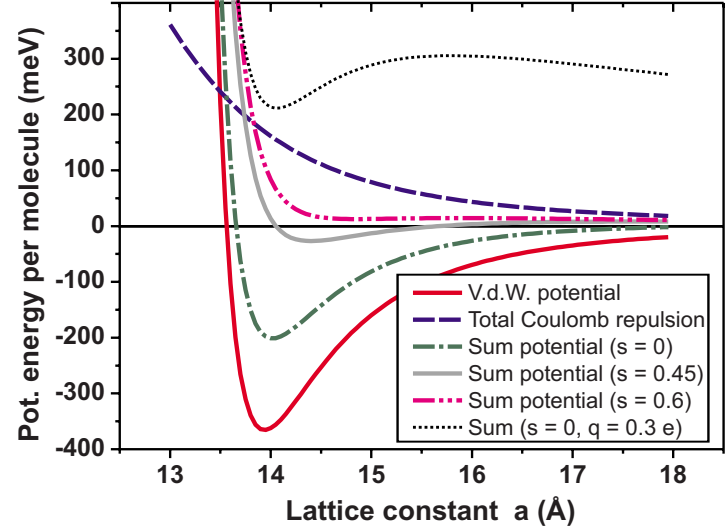

FIG. 5. (Color online) Different intermolecular potentials (per molecule) calculated in a closed HBC layer. The potential curves labeled "Sum" arise when adding the total Coulomb potential and the van der Waals potential. If $s>0$, the van der Waals potential is modified according to Eq. (1). The dotted black curve is calculated with an additional net charge $q$ on each HBC molecule.

by a factor of $\approx 3$ from the values in Fig. 3 , due to the six next neighbor molecules. In Fig. 5, the total Coulomb potential (dashed blue curve) is compared to the van der Waals potential (solid red curve) and to the sum of both, i.e., the total intermolecular potential according to the model discussed above (dash-dotted green curve). Clearly the observed repulsive behavior is not reflected by this total potentialenergy curve which is attractive over the whole latticeconstant range between 14 and $18 \AA$ A. Possible origins of this discrepancy will now be discussed one by one.

It is unlikely that the influence of the push-back dipole is largely underestimated in our model. Although the plate capacitor model used is of very basic nature, the overall repulsion could be explained only if the model underestimates the repulsive effect by a factor of 20 . Despite this huge deviation, such a strong influence would render the difference between, for example, PTCDA and HBC almost irrelevant which is, by no means, reflected in the experimental results.

The repulsion between the partial charges on neighboring HBC molecules should be reproduced rather accurately by the model used. Here, a factor of 3 would be necessary to explain the repulsion which would, in turn, mean a strong modification of the intramolecular charge distribution upon physisorption, which is completely implausible. It would also lead to an unrealistically large attraction in the case of PTCDA.

The existence of a hypothetical net charge on each molecule in the layer is, in principle, a way to explain the repulsion. While such a charge could emerge from a charge transfer from/to the substrate, only a very small partial charge, if any, is expected to be on the molecules due to this mechanism. The lowest unoccupied molecular orbital of the adsorbed HBC is not below the Fermi level $E_{\mathrm{F}}$ and the DOS at $E_{\mathrm{F}}$ is very small. ${ }^{1}$ Consequently, no (partial) filling of molecular orbitals is expected for $\mathrm{HBC}$ on $\mathrm{Au}(111)$. A small net charge on the molecules would, even if present, not be sufficient to explain the repulsion as shown for the case of a charge of +0.3 e. The respective dotted black curve in Fig. 5 implies, although at positive energies, still an attractive force in the region between 14.0 and $15.8 \AA$. 
As discussed in the last section, the force field model with OPLS parameters correctly reproduces the intermolecular potential in thick HBC films, and can thus not be the source of the huge discrepancy. In the case of molecules adsorbed directly and planar on a metal surface, however, the results obtained by this method might indeed differ significantly from the actual situation as the influence of a substrate is not included in the OPLS parameters. As the London forces responsible for the attractive part of the LJ potential result from polarization fluctuations on the molecules, they are subject to screening by the metal electrons which "compensate" the temporary dipoles. Several publications deal with the phenomenon of such a screening in case of adsorbed rare-gas atoms. ${ }^{32-34}$ As the considerations made in these papers are of universal character, the main ideas can most likely be transferred to larger adsorbates as well. While in Refs. 32 and 33 the remaining attraction is $\geq 2 / 3$ of the value in vacuum, Mahanty showed that an even stronger screening may occur if the molecules are partially "embedded" in the electron density spilling out of the metal surface. ${ }^{34}$ Then, the screening strength depends on the surface-plasmon frequency of the metal substrate, and a higher plasmon frequency leads to a stronger screening effect. As the lowest absorption frequency of HBC (about $2.8 \mathrm{eV}$ ) (Ref. 35) is in the range of the plasmon frequency of $\mathrm{Au}(2.5 \mathrm{eV}),{ }^{36}$ a considerable weakening of the molecule-molecule attraction should be expected. Although we are unable to give a precise value for the screening strength of the London forces (the model in Ref. 34 is too simple to be fully transferable) the screening can be accounted for by a parameter $s$ in the Lennard-Jones potential such that $s=0$ means no screening while $s=1$ would be a full screening of the London attraction,

$$
V_{m o l-m o l}=4 \epsilon\left\{\left(\frac{\sigma}{r}\right)^{12}-(1-s)\left(\frac{\sigma}{r}\right)^{6}\right\} \text {. }
$$

In Fig. 5 a comparison of the total potential for $s=0, s$ $=0.45$, and $s=0.6$ is made, revealing that only in the case of the strongest screening considered $(s=0.6)$, a potential curve is obtained that is repulsive in the whole lattice-constant range.

Here, a remark on the role of the entropy on the observed repulsion is necessary: it is practically impossible to include the free energy $F=U-T \cdot S$, rather than the internal energy $U$, into the theoretical considerations as the investigated system is far too complex to obtain an accurate value $\Delta S$ for the observed phase transition. Still, a possible influence can be roughly estimated to put it into perspective: under the assumption of an unscreened London attraction $(s=0)$, the potential-energy minimum shown in Fig. 5 (dashed-dotted green) has a depth of $200 \mathrm{meV}$ which has to be compared to $k T=25 \mathrm{meV}$. An entropic influence on the disorder can consequently be expected only in case of a significant screening, associated with a shallow minimum. Whether the real potential-energy curve is entirely repulsive for $\mathrm{HBC}$ or whether it is weakly attractive in a certain lattice-constant range cannot be answered completely.

\section{CONCLUSION}

We find a phase transition from a disordered isotropic distribution of HBC molecules on $\mathrm{Au}(111)$ existing at a coverage below $0.7 \mathrm{ML}$ to a highly ordered phase at higher coverage. The ordered phase exhibits a coverage dependent and thus tunable lattice constant. The intermolecular potential is modeled including the Coulomb as well as the van der Waals potential between the molecules. The results show that the local (push-back) interface dipole accounts only for a small part of the Coulomb interaction and is outweighed by the (in the case of HBC: repulsive) Coulomb forces between partial charges on neighboring molecules. We have shown as well that the repulsive nature of the total intermolecular potential can only be obtained by assuming a partial screening $(s \gtrsim 0.6)$ of the attractive London forces due to the presence of the metal substrate. Although the accuracy of this estimation is naturally limited by the simplicity of the model used, the occurrence of a considerable screening together with the lack of attractive Coulomb forces provides a plausible explanation not only for the case of HBC discussed here but also for the repulsion observed for other molecular species at sub-ML coverage.

Our analysis and conclusion is in a way complementary to the discussions of intermolecular repulsion in Refs. 18 and 19. Except for the push-back dipole, we discuss the charge distribution in the molecular plane, i.e., molecular multipole moments, as charge transfer is not expected to play an important role in the case of $\mathrm{HBC}$ on $\mathrm{Au}(111)$. In contrast, Refs. 18 and 19 focus on charge redistributions normal to the surface plane; i.e., local interface dipoles as the investigated molecules are chemisorbed on the surface. For these cases, the additional contribution of the intramolecular charge distribution (and of the "crosstalk," when the in-plane charge distribution is altered by the charge donation/backdonation) can only be estimated. At least for the phthalocyanine molecules, ${ }^{19}$ the contribution is expected to be repulsive due to the hydrogen atoms in the outer rim.

It turns out that $\mathrm{HBC}$ on $\mathrm{Au}(111)$ is perfectly suited as a starting point for the fabrication of organic-organic heterosystems as mentioned in the Introduction, since the repulsive potential allows the growth of $\mathrm{HBC} \mathrm{ML}$ domains with a substantially reduced density of defects, holes, grain boundaries or molecules in the second ML and, in addition, with a freely tunable lattice constant (between 14.2 and $\approx 16 \AA$ ).

\section{ACKNOWLEDGMENTS}

Financial support by Deutsche Forschungsgemeinschaft (DFG) Grants No. FR875/9 and No. FR875/11 is gratefully acknowledged. 
*http://www.iapp.de; wagner@iapp.de

${ }^{1}$ H. Proehl, M. Toerker, F. Sellam, T. Fritz, K. Leo, C. Simpson, and K. Müllen, Phys. Rev. B 63, 205409 (2001).

${ }^{2}$ F. Sellam, T. Schmitz-Hübsch, M. Toerker, S. Mannsfeld, H. Proehl, T. Fritz, K. Leo, C. Simpson, and K. Müllen, Surf. Sci. 478, 113 (2001).

${ }^{3}$ P. Ruffieux, O. Gröning, M. Bielmann, C. Simpson, K. Müllen, L. Schlapbach, and P. Gröning, Phys. Rev. B 66, 073409 (2002).

${ }^{4}$ L. Gross, F. Moresco, P. Ruffieux, A. Gourdon, C. Joachim, and K.-H. Rieder, Phys. Rev. B 71, 165428 (2005).

${ }^{5}$ H. Glowatzki, G. N. Gavrila, S. Seifert, R. L. Johnson, J. Räder, K. Müllen, D. R. T. Zahn, J. P. Rabe, and N. Koch, J. Phys. Chem. C 112, 1570 (2008).

${ }^{6}$ P. Ruffieux, K. Palotás, O. Gröning, D. Wasserfallen, K. Müllen, W. A. Hofer, P. Gröning, and R. Fasel, J. Am. Chem. Soc. 129, 5007 (2007).

${ }^{7}$ M. Treier, P. Ruffieux, R. Schillinger, T. Greber, K. Müllen, and R. Fasel, Surf. Sci. Lett. 602, L84 (2008).

${ }^{8}$ R. Forker, D. Kasemann, T. Dienel, C. Wagner, R. Franke, K. Müllen, and T. Fritz, Adv. Mater. 20, 4450 (2008).

${ }^{9}$ D. Kasemann, C. Wagner, R. Forker, T. Dienel, K. Müllen, and T. Fritz, Langmuir 25, 12569 (2009).

${ }^{10}$ N. Karl and C. Guenther, Cryst. Res. Technol. 34, 243 (1999).

${ }^{11}$ S. C. B. Mannsfeld, LEEDSIM available from sim4tec $\mathrm{GmbH}$ (www.sim4tec.com).

${ }^{12}$ C. Günther, Organische Molekularstrahlepitaxie: Ordnungsprinzipien großer Aromaten auf Schichthalbleitern (Logos Verlag, Berlin, 1998).

${ }^{13}$ I. Horcas, R. Fernández, J. M. Gómez-Rodríguez, J. Colchero, J. Gómez-Herrero, and A. M. Baro, Rev. Sci. Instrum. 78, 013705 (2007).

${ }^{14}$ L. Gao, Z. T. Deng, W. Ji, X. Lin, Z. H. Cheng, X. B. He, D. X. Shi, and H.-J. Gao, Phys. Rev. B 73, 075424 (2006).

${ }^{15}$ C. Seidel, R. Ellerbrake, L. Gross, and H. Fuchs, Phys. Rev. B 64, 195418 (2001).

${ }^{16}$ S. Müllegger, I. Salzmann, R. Resel, G. Hlawacek, C. Teichert, and A. Winkler, J. Chem. Phys. 121, 2272 (2004).

${ }^{17}$ W. X. Huang and J. M. White, J. Phys. Chem. B 108, 5060 (2004).

${ }^{18}$ I. Fernandez-Torrente, S. Monturet, K. J. Franke, J. Fraxedas, N. Lorente, and J. I. Pascual, Phys. Rev. Lett. 99, 176103 (2007).

${ }^{19}$ C. Stadler, S. Hansen, I. Kröger, C. Kumpf, and E. Umbach, Nat. Phys. 5, 153 (2009).

${ }^{20}$ Hypercube, Inc., HYPHERCHEM (www.hyper.com).

${ }^{21}$ N. D. Lang and W. Kohn, Phys. Rev. B 7, 3541 (1973).

${ }^{22}$ S. Henze, O. Bauer, T.-L. Lee, M. Sokolowski, and F. Tautz, Surf. Sci. 601, 1566 (2007).

${ }^{23}$ P. C. Rusu, G. Giovannetti, C. Weijtens, R. Coehoorn, and G. Brocks, J. Phys. Chem. C 113, 9974 (2009).

${ }^{24}$ S. C. B. Mannsfeld, POWERGRID available from $\operatorname{sim} 4$ tec $\mathrm{GmbH}$ (www.sim4tec.com)

${ }^{25}$ S. C. B. Mannsfeld, K. Leo, and T. Fritz, Phys. Rev. Lett. 94, 056104 (2005).

${ }^{26}$ W. Damm, A. Frontera, J. Tirado-Rives, and W. L. Jorgensen, J. Comput. Chem. 18, 1955 (1997).

${ }^{27}$ N. Atodiresei, V. Caciuc, P. Lazic, and S. Blügel, Phys. Rev. Lett. 102, 136809 (2009).

${ }^{28}$ Here, the substrate has no influence and the respective energy solely stems from intermolecular interaction.

${ }^{29}$ R. Zacharia, H. Ulbricht, and T. Hertel, Phys. Rev. B 69, 155406 (2004).

${ }^{30}$ S. Mannsfeld, M. Toerker, T. Schmitz-Hübsch, F. Sellam, T. Fritz, and K. Leo, Org. Electron. 2, 121 (2001).

${ }^{31}$ I. Chizhov, A. Kahn, and G. Scoles, J. Cryst. Growth 208, 449 (2000).

${ }^{32}$ O. Sinanoglu and K. S. Pitzer, J. Chem. Phys. 32, 1279 (1960).

${ }^{33}$ A. D. McLachlan, Mol. Phys. 7, 381 (1964).

${ }^{34}$ J. Mahanty, Phys. Rev. B 35, 4113 (1987).

${ }^{35}$ W. Hendel, Z. Khan, and W. Schmidt, Tetrahedron 42, 1127 (1986).

${ }^{36}$ A. Pulisciano, S. J. Park, and R. E. Palmera, Appl. Phys. Lett. 93, 213109 (2008). 